\title{
Hybrid Monte Carlo simulations of vertical electronic transitions in acetone in aqueous solution
}

\author{
Anders Öhrn · Gunnar Karlström
}

Received: 27 April 2006 / Accepted: 1 September 2006 / Published online: 18 October 2006

(C) Springer-Verlag 2006

\begin{abstract}
A simulation of the $n \rightarrow \pi^{*}$ absorption and the $\pi^{*} \rightarrow n$ fluorescence of acetone in aqueous solution is reported. The model has an explicit solvent representation with an effective $a b$ initio treatment of the solute. The model attempts to balance quantum chemistry, intermolecular interactions and statistical thermodynamics. It includes a non-electrostatic perturbation on the solute which models the solute-solvent exchange repulsion and the restriction put on the electronic structure of the solute by the antisymmetry to the solvent. The solvent shift to the absorption transition is found to be between 0.16 and $0.21 \mathrm{eV}$; the shift to the fluorescence transition is found to be between 0.02 and $0.05 \mathrm{eV}$. The simulation supports the conclusion that the first peak in the fluorescence spectrum of acetone is from a single molecule in equilibrium with the solvent, not from an excimer.
\end{abstract}

Keywords Acetone $\cdot$ Explicit solvent model $\cdot$ Pauli repulsion $\cdot$ Excited state

\section{Introduction}

Much thanks to modern quantum chemical methods, a good deal of knowledge of electronically excited states, spectroscopy and photochemistry of molecules in gasphase is available today. This assortment of methods has progressed from work on the uncorrelated Hartree-Fock method to methods which include more electron correlation, and are nowadays available in highly optimized

A. Öhrn $(\varangle) \cdot$ G. Karlström

Department of Theoretical Chemistry, Chemical Centre,

Lund University, P.O. Box 124, 221 00, Lund, Sweden

e-mail: anders.ohrn@teokem.lu.se quantum chemical software [1-4]. For molecules in the liquid phase, however, theoretical methods have not evolved as far.

To approach this topic, two modes of adapting quantum chemistry to studies of the condensed phase are proposed at a first level of approximation. The conceptually simplest one is a cluster calculation: a small set of solvent molecules plus the solute molecule are treated as a supermolecule in an ordinary quantum chemical calculation. The other mode is the effective solvent model: an effective Hamiltonian operator is formulated for the solute only, with the influence of the solvent on the solute accounted for as a perturbation operator. The latter mode treats the solvent and the solute at different levels of sophistication, ranging from compact continuum representations of the solvent to the more elaborate explicit solvent description. This means that although the supermolecular approach can rely on the quantum chemical method to describe the solute-solvent interactions, the effective approach has to separately formulate the perturbation operator to take these interactions into account. This opens for a range of different effective solvent models.

Further, a condensed phase study has to also consider the non-zero temperature of the modelled system. Irrespective of how the solute-solvent interactions are described, it is also necessary under non-zero temperature conditions to consider the distribution of energy, or in other words, what effect the entropy has on the solvation. This aspect of quantum chemistry adapted to condensed phase studies requires some statistical thermodynamical treatment. Models with explicit solvent representation almost exclusively use a simulation method to numerically solve this problem. Consequently, all interactions or forces have to be evaluated for several 
configurations to obtain a representative sample of the system. To keep the computational effort within feasible bounds, the time needed for all calculations in a single configuration must not exceed rather strict limits.

In this study, calculations with an effective solvent model using an explicit solvent representation are presented. The model aims at striking a balance between the different but connected aspects of quantum chemical solvent models as delineated above. In other words, (1) the quantum chemical description of the solute, (2) the description of the solute-solvent interactions and (3) the statistical thermodynamics which in turn requires the inclusion of the solvent-solvent interaction into the consideration. The particular process studied is the $n \rightarrow \pi^{*}$ absorption and the $\pi^{*} \rightarrow n$ emission transition of acetone, $\left(\mathrm{CH}_{3}\right)_{2} \mathrm{CO}$, in aqueous solution. The model, called QMSTAT, has previously been used to study the same two transitions in formaldehyde in aqueous solution; the reader is also referred to these works for more details of the model than what is included in the summary model section $[5,6]$.

\section{Theoretical background}

Before the model is presented, a short theoretical background is given. In this section, some terminology is introduced and a few fundamental features of the influence of the solvent on electronic spectra are restated to prepare for subsequent arguments. For detailed account of this theory, see refs. [7-9].

Arguably, the most important sources of the shift relative gas-phase of absorption and fluorescence peaks in solution derive from the instantaneous modification of the solute-solvent intermolecular interaction. During the electronic transition the charge density undergoes a change and hence the intermolecular interaction is modified. Transitions of this type are much faster than nuclear relaxations. Consequently, only the electronic degrees of freedom (DOF) of the solvent are in equilibrium with the final state of the solute, whereas the nuclear DOF are frozen. The final state is thus a nonequilibrium state. Bayliss and McRae introduce the term Franck-Condon strain to denote this property of the final state [8]. To refine their theory Bayliss and McRae separate the Franck-Condon strain into two contributions: orientational strain and packing strain. The former strain refers to that the properties responsible for the mutual solute-solvent orientation can change due to the transition; these properties are almost exclusively related to the electrostatic interactions. The latter strain is connected with the sudden change of the short-range
Pauli repulsion between the solute and the adjacent solvent molecules.

Beside these considerations of the instantaneous transition that concern the solute-solvent interactions, there are also implications for the statistical thermodynamics of the system. The thermal average of a transition energy is

$\left\langle U^{*}-U\right\rangle=\frac{1}{N !} \int\left(U^{*}(\mathbf{r})-U(\mathbf{r})\right) n(\mathbf{r}) \mathrm{d} \mathbf{r}$

where $U(\mathbf{r})$ is the interaction potential for the initial state, $U^{*}(\mathbf{r})$ is the interaction potential for the final state and $n(\mathbf{r})$ is the $N$-particle nuclear distribution function for the initial state. The average, as well as the distributions, for the final state is thus computed with the particle nuclear distribution for the initial state [10]. According to the earlier discussion, the electronic DOF are on the other hand allowed to relax to the final state.

\section{Model}

How does the present solvent model, called QMSTAT, relate to the three connected aspects listed and explained in Sect. 1? The following discussion answers this question. Details are omitted and the reader is referred to previous publications $[5,6]$.

To start with, the third issue is about the statistical thermodynamical side of the problem. The key quantity in this respect is the distribution function $n(\mathbf{r})$. To obtain this quantity, the present model uses the exact Metropolis-Monte Carlo (MMC) simulation algorithm, which computes $n(\mathbf{r})$ with only statistical error $[11,12]$. It should be stressed that for the average transition energy, as defined in Eq. (1), to be exact, the initial state potential in the integrand has to be the same potential that defines the distribution. In practice this means that for the simulation in each step of the MMC algorithm the energy has to be computed using the same potential as when computing the transition energy distribution. In doing so, the present model emphasizes the statistical thermodynamical aspect of the solvation problem.

This leads to the first aspect: the quantum chemical method. Since these methods generally are computationally demanding, it is primarily with regard to this issue that the possibility of solving the exact statistical thermodynamical problem will be determined. Further, because the present model aims at a description of excited states in addition to ground states, the quantum chemical method needs to be able to describe these states. To start with the latter issue, the complete active space self-consistent field (CASSCF) method has shown to be a good choice in this respect [13-15]. The CASSCF 
method is, however, computationally demanding and involves several two-electron integral transformations. Therefore a more compact formulation of the wave function is used in QMSTAT with the aid of the CAS state interaction (CASSI) method [16,17]. The effective wave function of the solute in state $i$ in QMSTAT, $\Psi_{i}^{Q}$, is linearly expanded in a set of orthogonal eigenstates produced by CASSI. With this linear ansatz the variation method, to approximately solve the Schrödinger equation, involves diagonalizing the effective Hamiltonian matrix. The selection of CASSI states in the expansion can usually be made small and made to reflect the expected perturbation the solvent will exert on the solute; how this is specifically done for this system is described in Sect. 4.

Finally, to formulate the effective Hamiltonian the solute-solvent interaction has to be considered. The contribution from the electrostatic interaction between solute and solvent is included. Together with the frozen particle nuclear distribution function, $n(\mathbf{r})$, in the final state, this implies that the orientational strain in the non-equilibrium solvation of that state is accounted for. QMSTAT also models the packing strain by adding a non-electrostatic perturbation, $\widehat{V}_{\text {nel }}$, to the effective Hamiltonian. A matrix element of this operator scales as the overlap between solute and solvent raised to the power of two. The operator will raise the energy of configurations - nuclear as well as electronic - with large solute-solvent overlap. At very short-range $\widehat{V}_{\text {nel }}$ gives insufficient repulsion, so an additional term is added to the total energy. This term has a small effect on most thermally accessible configurations. The exact definition of this term has changed in comparison with previous publications and is presently for $\Psi_{i}^{Q}$

$$
\beta\left(\sum_{k \in \Omega} \sum_{l}\left\langle\Psi_{i}^{Q} \mid \psi_{l}^{k}\right\rangle\left\langle\psi_{l}^{k} \mid \Psi_{i}^{Q}\right\rangle\right)^{3}
$$

where $\psi_{l}^{k}$ is the $l^{\text {th }}$ occupied gas-phase solvent orbital on the $k^{\text {th }}$ solvent molecule, $\Omega$ the set of solvent molecules with a significant repulsion with the solute and $\beta$ is a parameter. Observe that this term does not contribute to the effective Hamiltonian, only to the total energy.

In determining the particle distribution $n(\mathbf{r})$ the solvent-solvent interaction is also of importance. In QMSTAT, the water molecules of the solvent are represented with four point-charges, three distributed polarizabilities and a Buckingham-potential for the dispersion and the short-range repulsion. This description has been designed to reproduce the ab initio water dimer interaction.[18] In the partitioning of the electro- static features into a permanent part (the point-charges) and a dynamic part (the polarizabilities), the present water potential overestimates the permanent part somewhat. Consequently, the fast component of the solvent response (from the electronic DOF), which is always in equilibrium with the solute, will be underestimated. We expect the resultant insufficient stabilization of the final state to be of comparable magnitude of the errors inherited in assumptions such as frozen water geometry and vertical transitions.

The dispersion interaction between solute and solvent is modelled with a distributed $1 / r^{6}$ expression. As an approximation, the dispersion interaction in the excited state is set equal to that in the ground state for a given configuration. Estimates of dispersion interactions in electronically excited states are difficult to make in explicit solvent models. In fact, it is easier to treat these effects in a macroscopic context; the question how well a macroscopic treatment applies to a microscopic problem remains, however (see discussion by Li et al. [19] on this subject for further details and references). In a polar solvent the electrostatic interactions are of greater significance, thus probably making the neglect of the contribution to shift from the dispersion interaction acceptable; in non-polar solvents this may no longer hold [20,21]. Repeated applications of the model will reveal if and when this assumption is tenable.

We conclude this section with a qualitative discussion of computational efficiency. The choice to solve the statistical mechanical problem exactly requires the present model to solve many similar quantum chemical problems. Most other explicit solvent models chose to sample solvent configurations with a simplified potential, and then take a statistically uncorrelated subset of these configurations and solve the quantum chemical problem for them. This is no longer an exact solution of Eq. (1). By spending some time before the simulation in constructing a compact orthogonal state basis, the quantum chemical method of the present model is far less computationally demanding than most other $a b$ inito and density-functional methods; thus rendering the present statistical mechanical approach feasible. The evaluation of the non-electrostatic operator involves computing several overlap integrals. There are efficient algorithms to do this and unless very large basis sets are used, the time required in this step is of comparable magnitude to the solution of the polarization equations. The continuum models, on the other hand, use both simpler statistical mechanical approach and simpler description of the solute-solvent interactions and can thus use more time on the quantum chemical problem. Now this makes them the only feasible way to add solvent effects to many quantum chemical problems. Exactly to how large 
systems the present method can be applied to in practice has so far not been gauged.

\section{Simulation protocol}

The input CASSCF states to the construction of the CASSI basis for the QM-region, in which only acetone is situated, are prepared as follows: the active orbitals are the occupied $\pi$, oxygen $n$ and anti-bonding $\pi^{*}$. The state-average CASSCF algorithm is used which optimizes with respect to the arithmetic average energy of the singlet ground state $S_{0}$ and the first singlet excited state $S_{1}\left(n, \pi^{*}\right)$. This small active space gives a sufficient account of the static correlation, whereas the dynamic correlation is poorly represented. It is well-known that to obtain good electronic transition energies a CAS second-order perturbation (CASPT2) calculation is needed $[22,23,3]$. To compute the solvent shift to the transition energy, however, the largest part of the error in the insufficient account of the dynamic correlation is canceled since it is present in both gas-phase and solutephase. An atomic natural orbital (ANO) basis set is used with a $10 \mathrm{~s} 6 \mathrm{p} 3 \mathrm{~d} / 7 \mathrm{~s} 6 \mathrm{p} 3 \mathrm{~d}$ contraction for carbon and oxygen, and a 7s3p/4s3p contraction for hydrogen [24]. First, a total of seven CASSCF calculations are performed: six with an electric field of magnitude 0.003 a.u. directed along each of the three axes in both positive and negative direction, and one calculation with no perturbation applied. These states are used as input to CASSI which produces a total of 14 states (two states for each stateaverage CASSCF calculation). This set will be called the electric field set (EFS) in subsequent sections. Another larger CASSI basis is also constructed: a total of 19 CASSCF calculations are performed including the same 7 calculations as above, but also 12 calculations with the 6 different Cartesian electric field gradients applied of magnitude 0.0003 a.u., both positive and negative; the origin of the electric field gradient is chosen as the centre of mass. In total 38 states are produced. In subsequent sections they are called the electric field and gradient set (EFGS). The structure of acetone is optimized with analytical state-average CASSCF gradients with the same basis set and active space as above for both ground and excited state [25]. The bent structure of the excited state of acetone is thus obtained [26-28].

The parameters for the dispersion interaction between solute and solvent are fitted to an intermolecular second-order perturbation calculation to a single determinant wave function. The two parameters in the repulsive energy terms are fitted to reproduce a counterpoise corrected supermolecular CASPT2 pair-potential between water and acetone [29]. Parameter values are
Table 1 Parameters to the QMSTAT potential

\begin{tabular}{llll}
\hline & & $n \rightarrow \pi^{*}$ & $\pi^{*} \rightarrow n$ \\
\hline Repulsion & $d$ & -0.35 & -0.37 \\
& $\beta$ & 5.0 & 7.0 \\
& $\Omega$ Cut-off & 10.0 a.u. & 10.0 a.u. \\
Dispersion & $D_{C, O}$ & 72.5 & 72.5 \\
& $D_{C, H}$ & 0.4 & 0.4 \\
& $D_{O, O}$ & 65.7 & 65.7 \\
& $D_{O, H}$ & 0.4 & 0.4 \\
& $D_{H, O}$ & 0.4 & 0.4 \\
& $D_{H, H}$ & 0.0 & 0.0 \\
Field damp. & $\alpha$ & 0.5 & 0.5
\end{tabular}

All relevant equations are defined in [5]; the parameters to the water-water potential are listed in [18]

included in Table 1. In the parameterization of the higher order repulsive term, errors from the limitations in the simplified expression for the interaction are to some extent canceled in order to reproduce the supermolecular potential. The difference in the value of $\beta$ between ground and excited state is therefore only a sign that the errors in the description of the interactions are different for the two states.

Every hundredth of a total of $2.4 \times 10^{6}$ Monte Carlo steps is sampled to construct $n(\mathbf{r})$ for each CASSI-basis and transition. The simulation is performed under constant temperature (298K) and pressure (1 atm.) with 100 explicit solvent molecules which together with the solute is contained in a spherical dielectric cavity of variable radius. For practical details of the simulation procedure in a cavity, see refs. [5,30].

For all quantum chemical calculations, the MOLCAS quantum chemical program package is used [31].

\section{Results and discussions}

\subsection{Absorption: $n \rightarrow \pi^{*}$}

The solvent shift distributions for acetone in water with the two different CASSI basis sets are shown in Fig. 1. The average shifts for EFS and EFGS are 0.16 and $0.21 \mathrm{eV}$, respectively, where a positive number means that the transition has been shifted to shorter wavelengths (blue shifted).

The difference between the curves in Fig. 1 does not come from a difference in response properties of acetone in the two basis sets. An analysis of the solute response distribution reveals that the two CASSI bases have (1) similar dipolar response, and only (2) different quadrupolar response, with the EFGS basis set having a wider distribution. Both results are expected from the construction of the basis sets. 


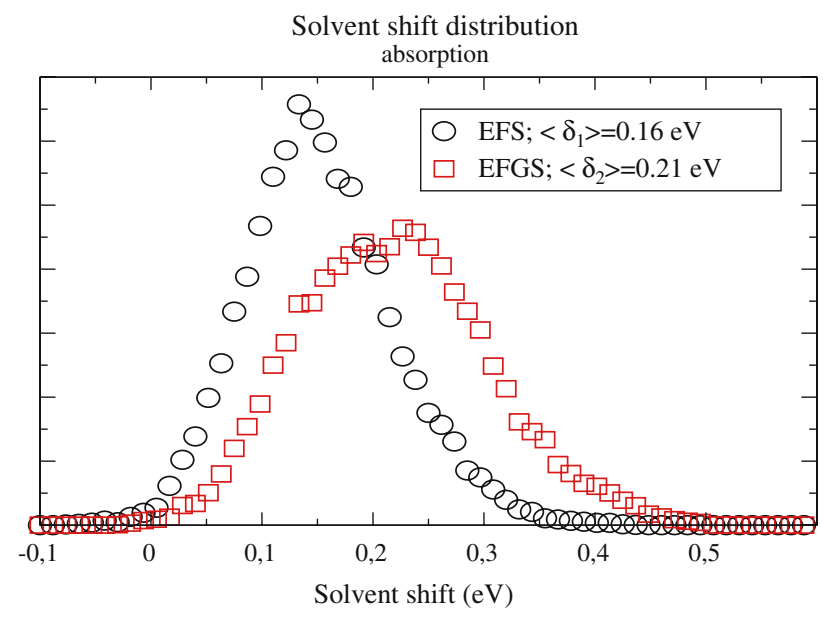

Fig. 1 Solvent shift distribution for the vertical $n \rightarrow \pi^{*}$ absorption of acetone in aqueous solution for both EFS and EFGS basis; averages are 0.16 and $0.21 \mathrm{eV}$, respectively

The contribution from the solute-solvent repulsion to the shift is shown in Fig. 2. The correlation is between the shifts in Fig. 1 and the shifts obtained with all repulsive parameters set to zero. For the smaller EFS basis, there is almost without any exception a greater blue shift with than without a solute-solvent repulsion. For the EFGS basis there are more configurations that are shifted to longer wavelengths by the packing strain, but the majority is oppositely shifted. The simple picture of the packing strain as an increased repulsive interaction between the solute and solvent in the vertically excited state can account for the points above the 1:1-line. There is of course also a possibility that the sudden change of molecular size can happen to transform the solute to better fit the solvent cavity. This situation contributes to a red-shift but is expected to be rare. However, the packing strain is more complex than this, because the repulsion and the solute polarization can couple. To see this, assume there is no restraint put on the electronic structure through the non-electrostatic operator. Under such conditions, the solute will polarize to a charge density with an optimal interaction with the electrostatic features of the given solvent configuration. But that polarized density could involve a significant overlap with the solvent, which would bring about a penalty from the non-electrostatic operator if it had been active in the variational procedure. Therefore the solute will also polarize differently due to the coupling, and hence have a different electrostatic interaction, with the non-electrostatic operator present than without.

The difference between the EFS and the EFGS curve in Fig. 1 is to some extent explained by the difference between Fig. 2a and 2b. But Table 2 also indicates another possible source: both the permanent dipole and

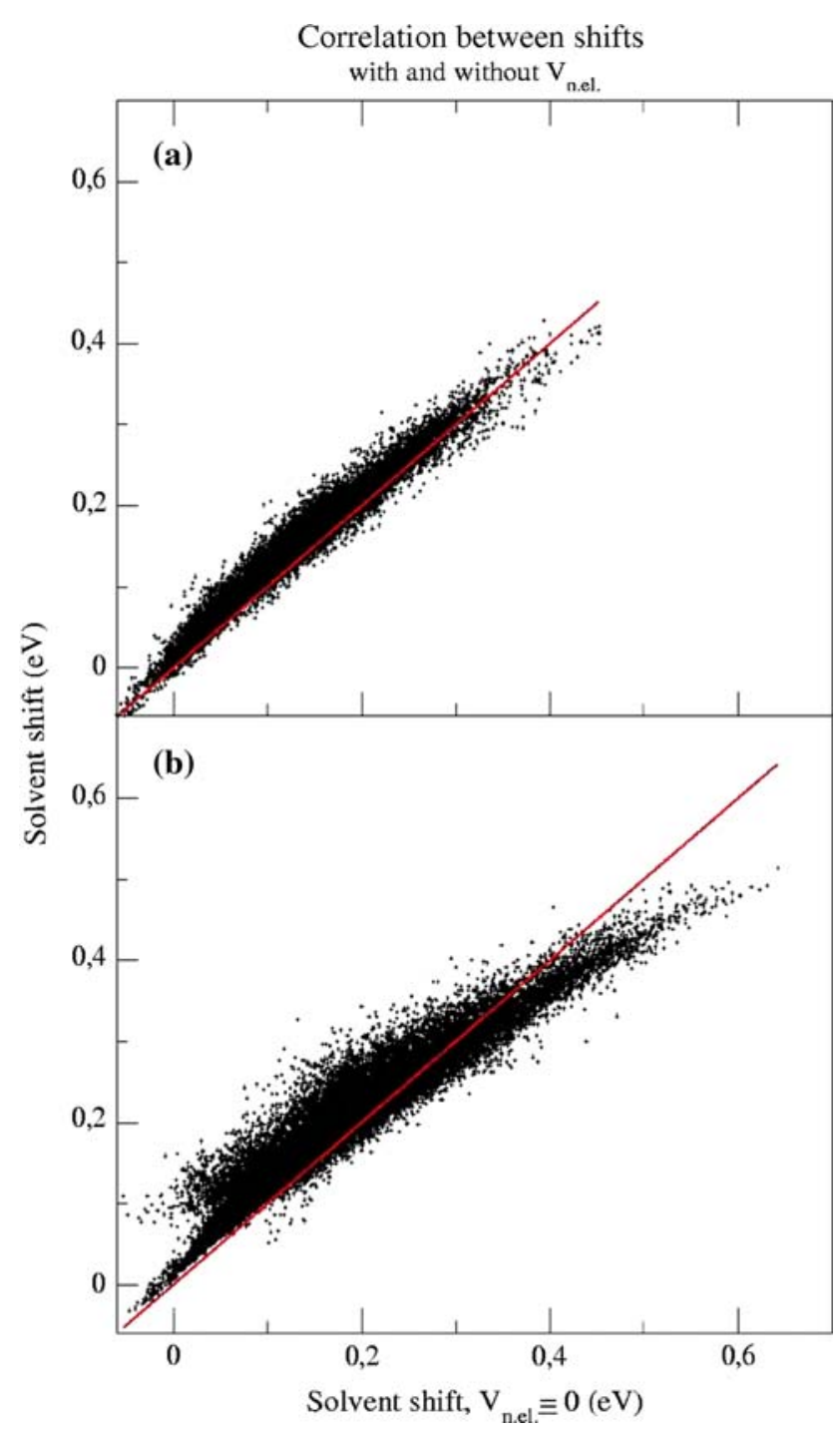

Fig. 2 Correlation between shifts for absorption with and without solute-solvent repulsion for the $\mathbf{a}$ EFS and $\mathbf{b}$ the EFGS basis. Points above the 1:1-line have greater shift with than without $V_{\text {nel }}$ active

quadrupole moment are different for the two basis sets. Two reasons are suggested to explain this phenomena. (1) With more states added to the basis set, not only will the way the solute responds to the solvent perturbation be altered, but also the intramolecular correlation: more states lead to more such correlation (see total energy in Table 2) and with that a slight modification of the charge density follows. (2) Since the CASSCF states that are input to CASSI overlap significantly, near linear dependencies can give rise to numerical instabilities in the ensuing algorithm. With more input states this potential problem is larger and more uncertainty is expected in the final density. Especially the greater reduction in quadrupole moment during the transition for the EFGS basis compared to the EFS basis suggests that part of 
Table 2 Solute properties of the ground $S_{0}$ and the vertically excited state $S_{1}^{*}\left(n, \pi^{*}\right)$, as well as the vertically de-excited state $S_{0}^{*}$ and the excited state $S_{1}\left(n, \pi^{*}\right)$ in gas-phase (atomic units)

\begin{tabular}{|c|c|c|c|c|c|}
\hline & & $S_{0}$ & $S_{1}^{*}\left(n, \pi^{*}\right)$ & $S_{0}^{*}$ & $S_{1}\left(n, \pi^{*}\right)$ \\
\hline \multirow[t]{2}{*}{$\left(\mu_{x}, \mu_{y}, \mu_{z}\right)$} & EFS & $(0 ; 0 ;-1.038)$ & $(0 ; 0 ;-0.506)$ & $(0 ; 0.277 ;-0.989)$ & $(0 ; 0.241 ;-0.665)$ \\
\hline & EFGS & $(0 ; 0 ;-1.051)$ & $(0 ; 0 ;-0.530)$ & $(0 ; 0.279 ;-0.989)$ & $(0 ; 0.241 ;-0.668)$ \\
\hline \multirow[t]{2}{*}{$|\mu|$} & EFS & 1.038 & 0.506 & 1.026 & 0.693 \\
\hline & EFGS & 1.051 & 0.530 & 1.027 & 0.696 \\
\hline \multirow[t]{2}{*}{$\left(\alpha_{x x}, \alpha_{y y}, \alpha_{y z}, \alpha_{z z}\right)$} & EFS & $(40.0 ; 31.2 ; 0.0 ; 41.8)$ & $(40.8 ; 32.0 ; 0.0 ; 39.0)$ & $(40.8 ; 33.0 ; 1.0 ; 41.6)$ & $(40.8 ; 34.4 ; 0.6 ; 37.4)$ \\
\hline & EFGS & $(40.0 ; 31.0 ; 0.0 ; 41.6)$ & $(41.0 ; 32.0 ; 0.0 ; 39.2)$ & $(40.6 ; 33.0 ; 1.0 ; 41.6)$ & $(40.8 ; 34.4 ; 0.4 ; 37.4)$ \\
\hline \multirow{2}{*}{$Q_{z z}$} & EFS & 2.98 & 2.22 & 3.67 & 2.73 \\
\hline & EFGS & 3.08 & 1.85 & 3.86 & 2.57 \\
\hline \multirow[t]{2}{*}{$\eta$} & EFS & 0.10 & 0.92 & 0.02 & 0.97 \\
\hline & EFGS & 0.12 & 0.91 & 0.13 & 0.89 \\
\hline \multirow[t]{2}{*}{ Total energy } & EFS & -192.0564 & -191.8896 & -192.0058 & -191.9296 \\
\hline & EFGS & -192.0571 & -191.8914 & -192.0063 & -191.9309 \\
\hline
\end{tabular}

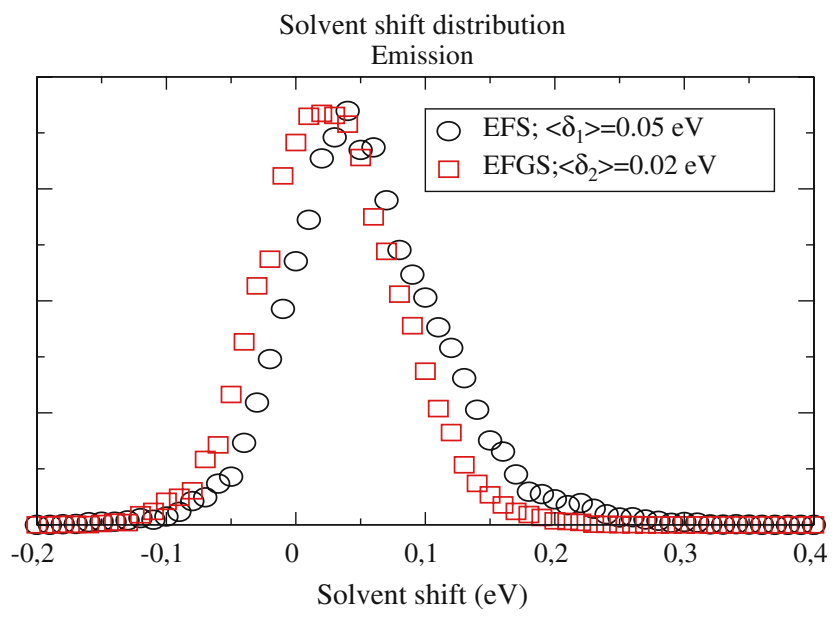

Fig. 3 Solvent shift distribution for the vertical $\pi^{*} \rightarrow n$ fluorescence of acetone in aqueous solution for both EFS and EFGS basis; averages are 0.05 and $0.02 \mathrm{eV}$, respectively

the larger blue shift originates from this dissimilarity in permanent moments.

\subsection{Emission: $\pi^{*} \rightarrow n$}

The solvent shift distributions for the two CASSI basis sets are shown in Fig. 3. There is on average a small blue-shift: $0.05 \mathrm{eV}$ for the EFS basis and $0.02 \mathrm{eV}$ for the EFGS basis. Because the final state is below the initial with respect to energy in an emission, a blue shift means that the vertically de-excited non-equilibrium ground state $S_{0}^{*}$ is more stabilized. Table 2 shows that the dipole moment in $S_{0}^{*}$ is larger than in $S_{1}\left(n, \pi^{*}\right)$. More favourable electrostatic interaction is, consequently, possible in the de-excited state, although the fact that the shift is so small shows that the particle distribution $n(\mathbf{r})$ is not in a such way that the optimal electrostatic interaction in the solvated $S_{0}^{*}$ is fully realized. This shows that from only solute properties the shift is non-trivial to estimate due to the non-equilibrium feature of the solvated final state.

The shifts to the fluorescence peak are not that different between the two basis sets. But the induced dipoles and quadrupoles have the same features as in the calculation on the absorption, i.e. the induced dipole distribution are almost the same, while the quadrupole distributions differ significantly.

The packing strain has a different effect on the shift for the emission than for the absorption. The points in Fig. 4 are clustered more evenly around the 1:1-line compared to Fig. 2, hence the packing strain contributes negligibly to the average shift. This agrees with the common notion that the size of a molecule in its excited state is larger than in the ground state. The solvent cavity has adapted to the size and shape of the excited state, and then when there is a de-excitation the smaller $S_{0}^{*}$ occupies the same cavity and hence experiences less repulsion. However, the change in shape of a molecule can, if the alteration of average size is not large enough, lead to increased repulsion. From the electric moments in Table 2 it is concluded that acetone undergoes at least some change in shape during the transition. Finally, the same mechanism that couples the non-electrostatic and electrostatic interactions in the absorption applies here as well, and it can act to further increase or decrease the shift.

As Table 2 shows the addition of more states to the basis causes a change in the permanent electric moments also for the two states relevant for the emission. Therefore, it is not obvious that the shifts computed with the EFS and the EFGS basis should be more similar for the emission than for the absorption. Since the total shift is smaller in the former transition than in the latter, it is likely, though, that also the difference in the shift between the two basis sets becomes smaller. In addition 


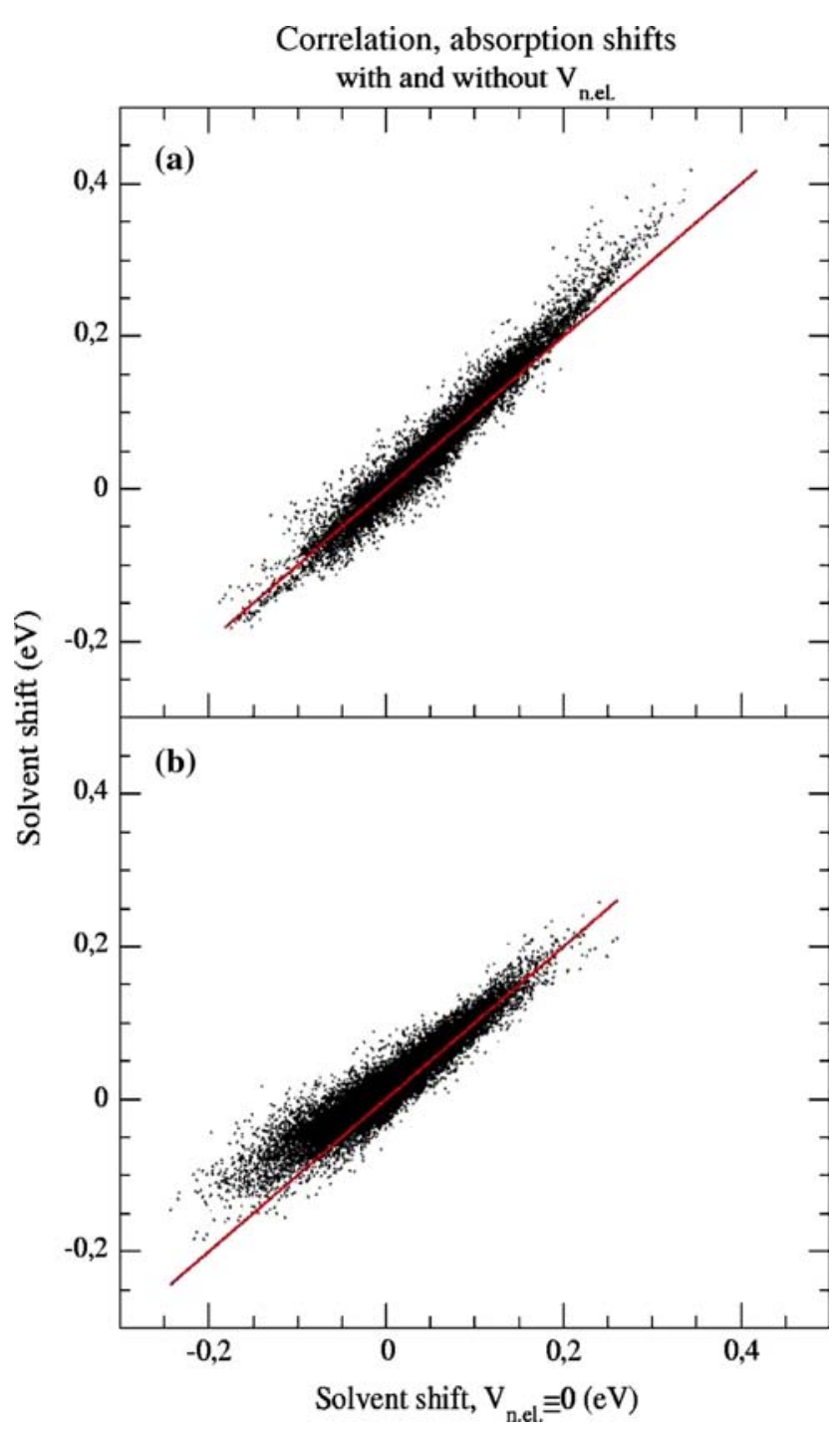

Fig. 4 Correlation between shifts for the emission with and without solute-solvent repulsion for the $\mathbf{a}$ EFS and $\mathbf{b}$ the EFGS basis. Points above the 1:1-line are blue shifted by the non-electrostatic operator

to this, there is of course the possibility of fortuitous cancellations: cancellations will occur if the modifications of the charge distribution for both states when adding more states to the basis are such that the alteration in electrostatic interaction during the transition happens to be very similar for both basis sets.

\subsection{Comparisons}

The experimental value for the shift to the $n \rightarrow \pi^{*}$ transition is in the range 0.19 to $0.21 \mathrm{eV}[32,33]$. The values refer to the energy with maximal extinction coefficient. The theoretical values refer to the average of the solvent shift distribution to the vertical transition with equal weight to all points. If the probability for the transition is constant over all frequencies and the vertical transition is the most probable transition, this difference between experiment and simulation is insignificant. It is an idealization to assume this, and any comparison between simulation and experiment will, to some extent, be ambiguous (see the work by Bernasconi et.al [34] for a study of the effect the solvent has on the intensity of the absorption). But these effects are not of such magnitude that a comparison is futile, and the simulated average solvent shift to the absorption transition of 0.16 and $0.21 \mathrm{eV}$ for the EFS and EFGS basis, respectively, are in agreement with the experiment.

There are several previous theoretical studies of acetone in aqueous solution in the literature using numerous different approaches with regard to the three issues in Sect. 1 [19,34-45]. The shifts of the previous studies are in the range 0.14 to $0.38 \mathrm{eV}$; see Table 7 of Aidas et.al. for a summary of all theoretical results up to 2005 [43]. The result of the present study belongs to the lower end of this interval.

The experimental determination and interpretation of the fluorescence spectrum of acetone in solution has not evolved without complications, and a short review of the early literature is justified. Borkman and Kearns studied the lifetime and quantum yield of acetone in various solvents, not water, however [46]. They made a number of relevant observations: (1) the fluorescence spectrum was found to be essentially independent of solvent, ranging from the non-polar hexane to the polar and protic methanol, with a peak at $3.0 \mathrm{eV}$; (2) the quantum yield was low and a lot of intersystem crossing occurred; (3) the singlet excited state life-time was estimated to be $2.5 \times 10^{-8} \mathrm{~s}$. From the absence of a solvent effect O'Sullivan and Testa interpreted the peak at $3.0 \mathrm{eV}$ as coming from an excimer, not an acetone monomer [47]. From experiments they concluded that the true monomer peak was solvent dependent and located at higher energies. But this conclusion was in turn contested by Renkes and Wettack, who from their own experiment found that impurities probably caused the additional peak of O'Sullivan and Testa [48]. Using carefully distilled solvents, including water, Renkes and Wettack reproduced the solvent independence of Borkman and Kearns with the fluorescence maximum at a slightly higher energy of $3.1 \mathrm{eV}$. The location of the peak showed no dependence on the acetone concentration in an interval of $2.5 \times 10^{-4}$ to $2.2 \times 10^{-2} \mathrm{M}$. The gas-phase fluorescence spectrum determined by Wettack was also found to have its peak at $3.1 \mathrm{eV}$ [49]. In a later article O'Sullivan and Testa conform to the interpretation that the medium unaffected peak at 3.0 or $3.1 \mathrm{eV}$ indeed belongs to the monomer [50]. To conclude, the solvent shift to the $\pi^{*} \rightarrow n$ fluorescence transition in (aqueous) 
solution is very small. The simulated shifts in the present study of 0.05 and $0.02 \mathrm{eV}$ thus agrees with experiment.

Fluorescence spectroscopy is harder to simulate and to our knowledge only two previous studies have done this for this system [40,42]. The reported value by Röhrig et.al. is $0.01 \mathrm{eV}$; they use a density functional QM/MM method with the wave function evolving according to the Car-Parinello scheme [42]. Coutinho and Canuto report a value of $0.23 \mathrm{eV}$; they use supermolecular cluster calculations with the semi-empirical INDO/CIS method [40].

The good agreement between experiment and simulation in this study, together with the similarity between acetone and formaldehyde, enables us to evaluate the previous QMSTAT study of the fluorescence of formaldehyde [6]. In that study a very small shift was also found. Since formaldehyde reacts with water, no experiment is available to compare with; hence a comparison with acetone was made. However, we falsely interpreted experimental data as a large blue-shift of $0.2 \mathrm{eV}$ or higher (an error also found in a table in the work by Röhrig et.al. [42]). With the present correct interpretation of experiment, the value of the shift for formaldehyde seems to be compatible with what the experiment is expected to give.

The results of the present simulations support the conclusion that the assumptions of the present model are fulfilled in the fluorescence experiment, namely (1) that the solute is in equilibrium with its surrounding and (2) can be approximated as being in infinite dilution. The short lifetime of the singlet excited state as reported by Borkman and Kearns makes the first condition not trivially fulfilled; and the second condition rules out that it is an excimer that fluoresces at $3.1 \mathrm{eV}$.

\section{Conclusions}

The QMSTAT model has been developed to be a balanced synthesis of quantum chemistry, intermolecular interactions and statistical thermodynamics. For a solvent model to be reliable this balance is necessary, we argue. In the present study the solvent shift to the $n \rightarrow \pi^{*}$ absorption transition and the $\pi^{*} \rightarrow n$ fluorescence transition of acetone in aqueous solution is simulated. Experiments are available for both transitions. The simulated results are judged to be consistent with the available experimental data.

The results support the conclusion that the singlet excited state is in equilibrium before it is transfered back to the ground state potential energy surface. Among experimentalists there has been some discussion whether the first fluorescence peak of solvated acetone originates from an excimer instead of a single acetone molecule in high dilution. Our results support the consensus interpretation that the relevant peak in fact comes from a single acetone molecule and not an excimer.

A model feature, which will require elaborations in future studies, is the construction of the CASSI basis. In the studies so far electric fields and electric field gradients have been used to obtain perturbed states to the basis. For small molecules with only one significantly polar group like formaldehyde and acetone, this construction has been adequate. For larger molecules with more extended chromophores (conjugated systems for example) this construction will hardly be adequate and local perturbation will be needed. The hazard of near linear dependencies will then become greater and some reformulation of the scheme is required.

\section{References}

1. Szabo A, Ostlund NS (1989) Modern quantum chemistry. Introduction to advanced electronic structure theory. Dover Publications, Mineola

2. Bauschlicher CW, Langhoff SR (1991) Chem Rev 91:701

3. Roos BO, Andersson K, Fülscher MP, Malmqvist P-Å, Serrano-Anderés L, Pierloot K, Merchán M (1996) Adv Chem Phys 93:219

4. Serrano-Andrés L, Merchán M (2005) J Mol Struct Theochem 729:99

5. Öhrn A, Karlström G (2006) Mol Phys (in press)

6. Öhrn A, Karlström G (2006) J Phys Chem A 110:1934

7. Reichhardt C (2003) Solvents and solvent effects in organic chemistry, 3rd edn. Wiley, Weinheim

8. Bayliss NS, McRae EG (1954) J Phys Chem 58:1002

9. Ågren H, Mikkelsen KV, (1991) J Mol Struct Theochem 234:425

10. Pimentel GC (1957) J Am Chem Soc 59:3323

11. Metropolis NA, Rosenbluth W, Rosenbluth MN, Teller AH, Teller E (1953) J Chem Phys 21:1087

12. Allen MP, Tildesley DJ (1989) Computer simulation of liquids. Oxford University Press, Oxford

13. Roos BO, Taylor PR, Siegbahn PEM (1980) Chem Phys 48:157

14. Roos BO (1987) Adv Chem Phys 69:399

15. Roos BO, Andersson K, Fülscher MP (1992) Chem Phys Lett 192:5

16. Malmqvist P- $\AA$ (1986) Int J Quantum Chem 30:479

17. Malmqvist P-Å, Roos BO (1989) Chem Phys Lett 155:189

18. Wallqvist A, Ahlström P, Karlström G (1990) J Phys Chem 94:1649

19. Li J, Cramer CJ, Truhlar DG (2000) Int J Quantum Chem 77:264

20. Longuet-Higgins HC, Pople JA (1957) J Chem Phys 27:192

21. Rösch N, Zerner MC (1994) J. Phys. Chem. 98:5817

22. Andersson K, Malmqvist P-Å, Roos BO, Sadlej AJ, Wolinski K (1990) J Phys Chem 94:5483

23. Andersson K, Malmqvist P-Å, Roos BO (1992) J Chem Phys 96:1218

24. Pierloot K, Dumez B, Widmark P-O, Roos BO (1995), Theor Chim Acta 90:87

25. Stålring J, Bernhardsson A, Lindh R (2001) Mol Phys 99:103

26. Laane J, (1999) In: Laane J, Takahashi H, Bandrauk AD (eds) Structure and dynamics of electronic excited states. Springer, Berlin Heidelberg New York, p 3 
27. Angeli C, Borini A, Ferrighi L, Cimiraglia R (2005) J Mol Struct Theochem 718:55

28. Walsh AD, (1953) J Chem Soc 2306

29. Boys SF, Bernardi F (1970) Mol Phys 19:553

30. Moriarty NW, Karlström G (1996) J Phys Chem 100:17791

31. Karlström G, Lindh R, Malmqvist P-A, Roos BO, Ryde U, Veryazov V, Widmark P-O, Cossi M, Schimmelpfennig B, Neogrady P, Seijo L (2003) Comput Mater Sci 28:222

32. Bayliss NS, McRae EG (1954) J Phys Chem 58:1006

33. Balasubramanian A, Rao CNR (1962) Spectrochim Acta 18:1337

34. Bernasconi L, Sprik M, Hütter J (2003) J Chem Phys 119:12417

35. Ten-no S, Hirata F, Kato S (1994) J Chem Phys 100: 7443

36. Thompson MA (1996) J Phys Chem 100:14492

37. De Vries AH, van Duijnen PTh (1996) Int J Quantum Chem 57:1067

38. Serrano-Andrés L, Fülscher MP, Karlström G (1997) Int J Quantum Chem 65:167
39. Martín ME, Sánchez ML, Olivares del Valle FJ, Aguilar MA (2000) J Chem Phys 113:6308

40. Coutinho K, Canuto S (2003) J Mol Struct Theochem 632:235

41. Aquilante F, Cossi M, Crescenzi O, Scalmani G, Barone V (2003) Mol Phys 101:1945

42. Röhrig UF, Frank I, Hutter J, Laio A, VandeVondele J, Rothlisberger U (2003) ChemPhysChem 4:1177

43. Aidas K, Kongsted J, Osted A, Mikkelsen KV, Christiansen O (2005) J Phys Chem A 109:8001

44. Crescenzi O, Pavone M, De Angelis F, Barone V (2005) J Phys Chem B 109:445

45. Neugebauer J, Louwerse MJ, Baerends EJ, Wesolowski TA (2005) J Chem Phys 122:094115

46. Borkman RF, Kearns DR (1966) J Chem Phys 44:945

47. O'Sullivan M, Testa AC (1968) J Am Chem Soc 90:6245

48. Renkes GD, Wettack FS (1969) J Am Chem Soc 91:7514

49. Wettack FS (1969) J Phys Chem 73:1167

50. O'Sullivan M, Testa AC (1970) J Am Chem Soc 92:5842 